\title{
Subcortical syntax: Reconsidering the neural dynamics of language
}

Elliot Murphy ${ }^{1,2^{\star}}$, Koji Hoshi ${ }^{3}$, Antonio Benítez-Burraco ${ }^{4}$

1. Vivian L. Smith Department of Neurosurgery, McGovern Medical School, University of Texas Health Science Center at Houston, Texas, USA

2. Texas Institute for Restorative Neurotechnologies, University of Texas Health Science Center at Houston, Texas, USA

3. Faculty of Economics, Keio University, Tokyo, Japan

4. Department of Spanish, Linguistics, and Theory of Literature (Linguistics), Faculty of Philology, University of Seville, Seville, Spain

*Corresponding author: Elliot Murphy (elliot.murphy@uth.tmc.edu)

Abstract: Subcortical contributions to core linguistic computations pertaining to syntax-semantics remain drastically under-studied. We critique the cortico-centric focus which has largely accompanied research into these higher-order linguistic functions and suggest that, while much remains unknown, there is nevertheless a rich body of research concerning the possible roles of subcortex in language. Although much current evidence emerges from distinct domains of cognitive neuroscience, in this review article we attempt to show that there is a clear place for subcortex in models of natural language syntax-semantics, including a role in binary set-formation, categorized object maintenance, lexico-semantic processing, morphosyntactic linearization, and cross-cortical representational integration. In particular, we consult models of language processing relying on oscillatory brain dynamics in order to investigate both the apparent and possible functional roles of subcortex in language.

Keywords: subcortex, language, basal ganglia, thalamus, hippocampus, syntax, semantics

Word count: 8,505

Figures: 1

Competing interests: The authors declare no competing interests. 


\section{Introduction}

Investigating the neural basis of natural language syntax and semantics (i.e., the generative, interpretive component of language) is one of the most important issues in modern cognitive neuroscience. It has also proven one of the most intractable directions of inquiry. Of those neurophysiological models which have succeeded in developing a considerable degree of descriptive scope (Friederici 2017, Griffiths et al. 2013, Wilson et al. 2011), these appear "cortico-centric" (Parvizi 2009), sidelining contributions from subcortex, despite Lenneberg's (1967) caveat about the likely importance of subcortex. In the past, and given the overwhelming focus of most neuroimaging research and the spatiotemporal resolution of traditional imaging techniques, this was justifiable and empirically motivated. We will here argue that this is no longer the case, and that there is increasing evidence from a broad range of domains suggesting that certain subcortical structures play important roles in natural language processing.

While subcortical structures have often been derided as the reptilian brain, responsible for only primitive drives, far removed from the higher echelons of thought, there is renewed motivation to re-situate subcortical regions like the thalamus and basal ganglia at the heart of language processing. The classical physician Galen held that the seat of the mind was in the ventricles, since donkeys also had highly convoluted brains. Through examining the crucial role of subcortical structures like the thalamus and basal ganglia in language, the Broca-Wernicke-Lichtheim model may go the way of the ventricular doctrine and the reptilian brain model, paving the way for more comprehensive accounts. And while neocortical expansion is the major focus in studies concerning the evolution of human intelligence, it is notable that the basal ganglia, cerebellum and hippocampus are two-to-three times larger in humans than in great apes (Gibson \& Jessee 1999). During the process of domestication, limbic structures are typically reduced, yet in humans limbic structures appear larger, not smaller (Bruner \& Gleeson 2019), rendering it unwarranted to sideline them when discussing human-specific higher cognitive capacities (Benítez-Burraco 2021).

Framing this discussion, we will here be assuming a particular approach to natural language phrase structure processing that we have developed in recent years, based on the central importance of neural oscillations and their interactions (Benítez- 
Burraco et al. 2017, 2018, Benítez-Burraco \& Murphy 2016, 2019, Hoshi 2017, 2019, Hoshi \& Miyazato 2016, Murphy 2015, 2016, 2018, 2020, Murphy \& Benítez-Burraco 2016, 2017, 2018, Progovac \& Benítez-Burraco 2019, Wilkinson \& Murphy 2016). Our goal is to explore the role of subcortical brain regions in this framework and to consult more recent developments in the field, marshalling further theoretical and empirical work which might expand and refine our understanding of language with respect to subcortical contributions. Our intention is not to provide a comprehensive review of subcortical contributions to all studied components of language; rather, we aim to provide a focused discussion of syntax-semantics. We also do not aim to make any particularly strong claims about the causal-explanatory role of subcortical structures in elementary linguistic computations, since we still lack the necessary empirical work to evaluate this. Yet, we nevertheless believe there to be ample evidence that conspires to suggest potential involvement of subcortical structures in a number of core components of natural language.

Lastly, we wish to present our discussion within the context of the following remark: Few neurolinguistic models of language seem to be exclusively committed to cortico-centrism, and the current absence of subcortex from many models does not necessitate the conclusion that the authors of these models wish to intentionally exclude subcortical structures, or otherwise deny their importance. While the notion that subcortical structures contribute to language remains, in our view, a minority position, there are nevertheless a range of researchers who appear to be converging on the view that subcortex is recruited for language; for instance, in bilingualism (Bice et al. 2020, Burgaleta et al. 2016, Cargnelutti et al. 2019, Hervais-Adelman et al. 2018, Stocco et al. 2014), phonological processing (Booth et al. 2007), syntax (Lieberman 2006, 2015), reading (Braun et al. 2019, Yeatman \& White 2021) and lexical access (Crinion et al. 2013, Hernandez \& Li 2007, Meinzer et al. 2006). Our aim is not to label various models as subcortical-inclusive or subcortical-exclusive, but to evaluate the apparent roles of subcortical structures in higher-order language processing, with particular emphasis on brain dynamics.

\section{Subcortical dynamics of language: Basal ganglia as syntactic generator}


Far from being isolated to inferior frontal regions or posterior temporal regions, subcortical grey matter structures such as the striatum and thalamus appear to be implicated in the processing of syntactic operations (Moro et al. 2001, Teichmann et al. 2015, Wahl et al. 2008). We will begin by reviewing the basal ganglia, before turning to other subcortical structures such as the thalamus, hippocampus and cerebellum.

The idea that subcortex plays a role - potentially a crucial role - in higher language functions is not a new one. Petersson et al. (2012) critique the classical Broca-Wernicke-Lichtheim language model of the brain by observing that the language network extends to substantial parts of superior and middle temporal cortex, inferior parietal cortex, along with subcortical areas such as the basal ganglia (Balari \& Lorenzo 2013) and hippocampus (Kepinska et al. 2018). Going beyond this, the potential interplay between subcortex and certain cortical structures involved in language has been noted, with language being a clear example of a network phenomenon, rather than a "localizationist" process ( $\mathrm{Li}$ et al. 2020). BA 44 and the posterior superior temporal cortex seem to be implicated in a pathway which supports core syntactic computations (Friederici et al. 2006, Poeppel 2014), but the precise role of any subcortical connectivity has been studied much less than these discrete centers of cortical activity during language processing.

One of the first modern models of language processing that proposed a central role for subcortical structures was Ullman's declarative/procedural model of the lexicon and grammar (Ullman 2001). In brief, the lexicon and the grammar are hypothesized to rely on two different memory systems; namely, associative memory, with distributed representations subserved by temporal lobe circuits, and procedural memory, subserved by frontal/basal ganglia circuits and specialized for computing hierarchical structures. A more elaborated example of how subcortex has been implicated in language is Lieberman's (2006) Basal Ganglia Grammar model, which proposes the existence of a pattern generator whose excitation/inhibition mechanism is in the basal ganglia. Recent work suggests that this might interface with working memory resources located in Broca's area and other portions of middle frontal gyrus (Santi et al. 2015). Lieberman estimates that the dorsolateral prefrontal circuit is involved in sentence comprehension, projecting from the prefrontal cortex towards the lateral dorso-medial region of the globus pallidus, and the thalamus, projecting back to the 
prefrontal cortex. Balari and Lorenzo (2013: 100-102) have gone somewhat beyond this and suggest that the basal ganglia-thalamic-cortical loop may be the circuit used as language's computational system operating within a structure of working memory networks.

As mentioned, we also aim to discuss the role of subcortex within the context of brain dynamics, not just connectivity or local cortical activation profiles. In terms of electrophysiological dynamics, we will assume the model in Murphy (2020) in which elementary syntactic combinatorics recruits a parahippocampal and cortico-basal ganglia-thalamo-cortical loop (i.e., some version of linguistic feature conjunction) coupled with posterior superior temporal gyrus/sulcus (syntactic categorization, or "labeling"), which is later coupled with left inferior frontal regions such as BA 44 and BA 45 which act as crucial memory buffers for the maintenance of hierarchically organized syntactic objects. For example, and following on from some of the empirical work mentioned above, this model proposes that when $\mathrm{Y}$-itemised linguistic representations (referring to mid- and broadband high-gamma neural activity) are coupled to parahippocampal $\theta$, some of them ultimately slow to $\beta$ to be maintained in short-term memory as the existing cognitive set (see Engel \& Fries 2010 for evidence that $\beta$ is responsible for such maintenance operations, and also Armeni et al. 2019 for evidence that unexpected words lead to $\beta$ decreases, disrupting the maintenance of the set). Evidence that this process is neurophysiologically plausible comes from Gollo et al. (2017), who showed that after cortical regions are coupled together, their frequency changes as a result of network interactions, such that "slower hub regions speed up, and faster peripheral regions slow down their activity". Moreover, the hippocampus is highly effective at "generating ordinal cell assembly sequences relevant to the particular situation", be that a spatial, temporal, auditory or memoryrelated function (Buzsáki \& Llinás 2017: 484). The domain-generality of its computational power indicates a likely crucial role in linguistic feature-set combinatorics, even if current empirical work concerning language and hippocampus seems to be limited to hierarchical structure processing (Blank et al. 2016) and semantics (Piai et al. 2016).

Keeping with these low frequency dynamics, findings reported in Gehrig et al. (2019) suggest a particular role of $\beta$ in phrasal categorization. These authors investigated speech memory representations using direct, intracranial cortical 
recordings in the left perisylvian cortex during delayed sentence reproduction in patients undergoing awake tumor surgery. Based on patient memory performance, the phase of fronto-temporal $\beta$ appears to represent sentence identity in working memory. The notion of sentential identity presupposes a labeled structure (e.g., Complementizer Phrase, Tense Phrase, Verb Phrase), seemingly represented (at least partially) by fronto-temporal $\beta$. Since subcortex is known to modulate frontotemporal connectivity, this opens up future inquiry into how this process impacts language. More generally, accumulating evidence suggests that $\beta$ holds objects, whereas y generates them (Martin \& Ravel 2014). Dean et al. (2012) relatedly show how $\beta$ is a good candidate for comparing old and new information from distinct modalities due to its wider temporal windows, likely drawing on different conceptual representations.

Low frequency dynamics are clearly relevant to syntactic-semantic computations, but what is the connection with subcortex? We will review below how basal ganglia is a primary generator of cortical $\beta$ waves. These dynamical considerations were already suggested in early work: For instance, in Kotz et al. (2003) it was reported that during the processing of sentences with verb-argument structure violations the resulting $\mathrm{N} 400$ (marking some form of late semantic-thematic integration process) was partially modulated by the basal ganglia. Friederici et al. (2003) also reported that syntactic violations in sentence processing not only resulted in greater activation levels in left anterior superior temporal gyrus and posterior frontal operculum, but also the putamen in the left basal ganglia.

The core position occupied by the basal ganglia in these explorations of natural language also fits well with imaging studies which have revealed the involvement of this region in 'syntactic complexity', specifically the processing of type-identity intervention of matching labels, being activated in an fMRI study when a noun phrase similar to the dependency head in a long-distance dependency intervenes in said dependency (Santi et al. 2015). This calls back to the notion of sentential identity. Indeed, the cortico-basal ganglia-thalamo-cortical loop is especially appealing with respect to its potential role in phrase structure building given the finding that different 'stations' on the loop can be responsible for information integration from distinct cortical sources: convergence appears to occur at the pallidum (Yelnik et al. 1984), subthalamic nucleus (Haynes \& Haber 2013) and thalamus (Theyel et al. 2010). These 
stations also display a degree of functional specificity, and it is possible that different features of linguistic representations are integrated into the phrase structure building process in a procedural manner. The real-time dynamics of this 'relaying' process are yet to be tapped by any direct, intracranial monitoring, to the best of our knowledge.

Reviewing related literature, Klostermann et al. (2013) conclude that "malfunction of the [basal ganglia] leads to deficits in applying combinatorial rules to linguistic messages, compatible with proposed superordinate [basal ganglia] functions, such as the sequencing or time-critical selection of input signals in general". Damage to thalamic relay stations of basal ganglia loops may also result in the typical constellation of monopitch, reduced stress and imprecise consonants (Ackermann \& Ziegler 2013, Duffy 2005), again indicating a role for these structure in aspects of temporality. Even though dorsal path connections may be implicated in constructing hierarchical phrase structures (Friederici 2017), other work suggests that the basal ganglia and thalamus act as network hubs forming a core circuit supporting large-scale integration (Acsády 2017, Bell \& Shine 2016); indeed, large-scale information integration is "a key computational priority" of the subcortex, for Bell and Shine. This model lends further weight to the idea that dorsal stream circuits can extend into subcortical regions to participate in maintenance/memory and syntactic categorization processes which are coordinated and structured by low frequency bands. While information segregation and storage is a clear priority for anterior temporal and posterior temporal regions, information integration appears to additionally recruit a complex system of subcortical structures - approximating closer the generative power of natural language.

In addition, deficits in syntactic rule application in Huntington's disease are connected to basal ganglia damage (Teichmann 2005). Huntington's disease patients exhibit normal performance in phoneme discrimination in the context of isolated words, but display a deficit in phrasal (syntactic) contexts (Teichmann et al. 2009), linking phrase-level computations to basal ganglia degeneration. Sambin et al. (2012) revealed that Huntington's disease patients with basal ganglia damage specifically exhibit syntactic deficits when processing Principle C (Binding Theory) pronoun violations, and not working memory deficits. 
The implementation of rule-based computations seems clear here, and it remains an open question whether language's rule-based grammatical processes recruit other subcortical structures. Current evidence at least points towards basal ganglia, but here another question arises: What 'rules' are engaged here? Recent work (Johari et al. 2019) suggests that, in Farsi-speaking Parkinson's disease patients, basal ganglia damage may not in fact be implicated in complex sentence comprehension, but rather executive functioning and, as the authors suggest, more minimal forms of syntactic computation involving "simpler rule-governed grammatical forms" (Johari et al. 2019: 232), as opposed to complex multi-phrasal working memory processing. A range of other work points to the role of the frontostriatal loop and basal ganglia in syntactic deficits in Parkinson's disease (for a review, see Smith \& Caplan 2018). As such, basal ganglia is recruited for minimal compositional schemes, although the full scope and format of these schemes remains an open area for research.

Moving beyond rule-based processes, are there any other related levels of linguistic computation seemingly relevant to subcortical activity? In light of the fact that the cognitive process of categorization is shared across vertebrates (Corballis 2002, Denenberg 1981, Mareschal et al. 2010, Rogers et al. 2013, Vallortigara et al. 1999), and the observation that the syntactic combinatorial process of MERGE and categorization share the property of recursive set-formation, Hoshi $(2018,2019)$ hypothesizes that MERGE as an unbounded recursive set-formation operation was phylogenetically derived from the cognitive process of categorization in our prelinguistic hominin ancestors. If categorization and syntactic combinatoriality are phylogentically related, it is possible that there are neural underpinnings common to both. In fact, as summarized in Lieberman (2006), categorization implicates interactions between the pre-frontal cortex and the striatum, as demonstrated in Huettel et al. (2002; see also Monchi et al. 2001). Seger and Miller (2010) note that categorization crucially implicates cortico-striatal loops, which may mediate interactions between fast reward-gated plasticity in the basal ganglia and slow plasticity in the cortex, enabling a balance between experiential learning and generalization.

Continuing with the theme of global coordination, top-down induced shifts in $\alpha$ phase between two cortical areas has been shown to strongly effect interregional $y$ coherence (Quax et al. 2017). In turn, it has been shown that this higher y coherence 
between cortical regions also results in more efficient transmissions of spiking information. These findings point to "the feasibility of a ... realistic mechanism for routing information in the brain based on coupled oscillations" (Quax et al. 2017). Given its prominent interareal $\alpha$ and its role in cross-cortical connectivity, the pulvinar would be able to carry out these combinatorial processes with great efficiency, allowing the brain to "enhance processing of uncued stimuli" (Quax et al. 2017), with the importance of "uncued stimuli" for neurolinguistics being clear given the - as classically formulated - creativity of grammar. Further, optogenetic approaches suggest that subgroups of the thalamic reticular nucleus facilitate attention switching from external stimuli to internal monitoring (Halassa et al. 2014), a dynamic process required, for instance, in the mapping of language-relevant sensory information to endogenous inferences. Saalman (2014) demonstrated that the envelope of cortical $Y$ power in areas V4 and TEO of the visual pathway was coupled to the phase of cortical $\alpha$ generated in higher-order thalamic nuclei, suggesting that the thalamus plays a major role in the creation of cortical $y$ rhythms known to be implicated in a variety of cognitive processes (via local ensemble activation). Since they have been shown to be involved in both local and global oscillatory control (Fogerson \& Huguenard 2016), the apparent flexibility of thalamic cellular and synaptic mechanisms could be readily exploited by language.

Moves towards direct intracranial monitoring of thalamic or subthalamic activity in humans during language processing (already a feasible goal, as demonstrated by Chrabaszcz et al. 2021) could further confirm or repudiate these ideas. While previous work has implicated subthalamic nucleus in speech production (Lipski et al. 2018), only recently has this region been implicated in lexical processing (Chrabaszcz et al. 2021), suggesting that the representational and computational scope of this and neighboring regions should be explored further.

Deepening our understanding of these regions, Deco et al. (2017) show that the reason why certain regions can oscillate at very low frequencies and sustain these rhythms is due to internal connectivity along with external thalamo-cortical connectivity, seemingly strengthening the ties between the thalamus and cortical language-relevant regions. Subcortex appears to play a causal role in coordinating the oscillatory behavior of cortical regions generating linguistic inferences. The kinds of low frequency entrainment and endogenous synchronicity in left fronto-temporal 
structures that is being connected to syntactic representations in recent literature (for review, see Meyer et al. 2020) are clearly a crucial part of any model of language, yet it may also be the case that without subcortex these fronto-temporal dynamics would not be possible. While we note that there are no cases of authors directly denying this possibility, there is also currently no direct evidence to support these dynamical systems-level proposals, with further empirical work jointly addressing neural dynamics and subcortical connectivity profiles being needed.

An additional reason for expecting research into subcortical language processing to be illuminating comes from the recent finding that human prefrontal cortex has the same relative volume of grey and white matter neurons as other primates, challenging any claims that uniquely human cognitive capacities arose due to an expansion of the prefrontal cortex (Gabi et al. 2016). Martin (2016) documents evidence suggesting that the classical view of the cortex as being exclusive to mammals is outdated, and that a number of vertebrates have cortex-like features.

How can we relate these ideas to more well-established findings of cortical involvement in syntax-semantics? Research by Matchin et al. (2017) considers the finding that damage to the inferior frontal gyrus does not impair basic structure-building processes and suggests that increased activity in this area during phrase structure building reflects the generation of top-down structural predictions. The inferior frontal gyrus and posterior superior temporal sulcus did not show increased activity for simple phrases in their fMRI experiment, in contrast for sentences, most likely because the simple phrases used did not trigger structural predictions. Frontal regions seem key to aspects of active inference needed to generate accurate syntax-relevant information - yet they may not be causally implicated in basic syntax-semantics. Mayberry et al. (2018) also investigated the brain of an individual who experienced minimal linguistic input until young adulthood, showing that during language processing the dorsal pathway in the right hemisphere was primarily active rather than the classical perisylvian network. In this connection, Martins and Villringer (2018) discuss how structured sequences that are difficult to process appear to implicate left inferior frontal gyrus, and not hierarchical structures specifically.

These results seem compatible with the claim that parts of the inferior frontal gyrus do not provide "the seat of syntax", but rather something of a footstool - a 
memory buffer and system of control interfacing with a construction memory stack in superior posterior temporal regions. The fact that the inferior frontal gyrus is active during the processing of unexpected stimuli suggests that its role is memory/controlrelated, not specifically hierarchy-related.

Going even further, Young et al. (2021) reviewed a range of existing results from intracranial cortical stimulating mapping and showed how left inferior frontal regions do indeed play a role in syntax-semantics, but likely in complex sentence processing, rather than in elementary structure building. Cortical stimulation mapping has also shown that action naming (involving verbs), which is more pertinent for basic syntactic structure than nominal information, recruits posterior temporal lobe sites, while common object naming (involving nouns) recruits more anterior temporal sites (Corina et al. 2005); in accord with what we have briefly reviewed about posterior temporal involvement in syntax (see also Flick et al. 2018 and Flick \& Pylkkänen 2020 for careful analysis and evidence for posterior temporal invovlement in minimal phrase binding).

With respect to any apparent human-specific computational properties of Broca's area and its memory buffer and externalization control system (i.e., its apparent involvement in processing linear morpho-syntactic relations, complex phonology, and its use as an additional syntactic workspace), Palomero-Gallagher and Zilles (2019) provide a tentative answer as to its cytoarchitectonic and microstructural uniqueness. These authors quantitively studied the cytoarchitecture of areas 44 and 45 using layerspecific grey level indices (volume proportion of neuropil and cell bodies) in serially sectioned and cell body stained human, bonobo, chimpanzee, gorilla, orangutan and macaque brains (studying homologues to areas 44 and 45 in non-human primates). Humans were found to have the largest neuropil volume, indicating greater space for local and interregional connectivity. This may play a causal role in the additional workspace demands needed by natural language syntax, but likely not in more elementary computations. Additional cortical stimulation mapping of elementary syntactic structures could speak to this hypothesis.

Other recent evidence points away from traditional cortico-centric models. Moreno et al. (2018) used fMRI to scan the brain of congenitally deaf adults who had acquired French sign language as their first language and written French as a second language while watching a list of signs which corresponded to syntactic constituents 
of varying sizes. They discovered an effect of constituent structure in the basal ganglia (including the head of the caudate and putamen) and a much smaller effect on temporal and frontal regions known to be involved in written language. When the same participants read sentences versus word lists, the processing of sentences resulted in activation in the basal ganglia. This study highlights the importance of the basal ganglia in modality-independent syntactic processing. A core part of natural language syntax (i.e., phrase categorization, labeling) may therefore take part in subcortical structures involving, and close to, the basal ganglia. In Murphy (2020), the categorization aspect of labeling was claimed to involve a network of posterior temporal regions and certain forms of cross-frequency coupling (and may also involve more direct involvement of the basal ganglia, and indeed other structures such as the hippocampus, as we will discuss below), while the maintenance/workspace aspects of labeling (which are necessary for any form of multi-phrasal construction; i.e., maintaining a category in memory), along with feature concatenation, more directly implicate subcortex. Supporting the centrality of this computation in natural language processing (as predicted in Hornstein 2009, Hornstein \& Pietroski 2009), there is recent evidence for the role of a category-sensitive phrase-building (labeling) mechanism driving the cortical tracking of hierarchical linguistic structures (Burroughs et al. 2021). We again return here to the possible role of subcortex in regulating and generating these forms of cortical oscillatory tracking mechanisms - a clear project for future research.

Pursuing this line of inquiry, left inferior frontal regions are regulated by the basal ganglia and thalamus via a cortico-basal ganglia-thalamic loop (Haber \& Calzavara 2009), an integrative system which is much more likely to be involved in structurebuilding operations than purely Broca's area alone. If anything, Broca's area seems specialized for executing retrieval operations which operate over constructed sets of linguistic features, hence why after a lesion to this area individuals retain the ability to comprehend and produce such feature-sets, albeit with reduced cognitive control over them. Contrary to standard models implicating the basal ganglia purely in physical movement, Haber and Calzavara (2009: 70) explore how it is also involved in "the processes that lead to movement" such as motivation and planning.

In connection with the important role of subcortical structures such as the basal ganglia and hippocampus in language processing, consider also Landau-Kleffner 
syndrome (LKS). This is a rare childhood neurological condition accompanied by loss of language skills and abnormal EEG signatures. Murugesan et al. (2020) demonstrated that the language delay in a patient with LKS is caused by pathology in the left side of the "language area" involving the basal ganglia and the hippocampus (and the temporal cortex). In particular, they discovered that the basal ganglia shows asymmetric diminished activity in the left hemisphere. While this study provides no means to causally separate the contributions of subcortical and cortical structures, basal ganglia and hippocampus at least appear implicated in language delay.

Other work suggests that disinhibiting a basal ganglia loop leads to the maintenance of a particular representation to the exclusion of others (Koziol et al. 2009), while the dependence on inhibitory rebound documented in $\beta 1$ (slow $\beta$ ) permits it to "continue in the absence of continuing input" (Kopell et al. 2010: 3). In humans, maintaining representations after "continuing input" may allow a labeled object to be held in memory beyond the perception of its immediate, inference-initializing elements.

What do these disparate findings about subcortex ultimately amount to, when faced with the much more robust, replicated findings about, say, the sensitivity of Broca's area and posterior temporal regions to various linguistic structures? We are not aiming to throw the baby out with the bathwater - on the contrary, we are interested in potential forms of reconciliation and providing further causal-explanatory details to the full range of brain dynamics of natural language, rather than isolating some subregion generally sensitive to linguistic structure. Alongside its putative role in feature concatenation and object maintenance, we propose that the basal ganglia could also be involved in the sequencing of syntactic information, likely a very early stage of externalization or the interface mapping from narrow syntax to external performance systems. Some linguists have proposed that aspects of syntactic computation could in principle relate to notions of linearity (e.g., Mathieu 2016, Richards 2016, but see Chomsky 2020, Chomsky et al. 2019), such that morphophonological factors may play a direct role in syntax. For instance, metrical requirements of affixes and other sensorimotor constraints are said to impact how syntactic computation is carried out. These stages of computation could potentially be encoded via the presently proposed neural dynamics of subcortex alongside functional connectivity with inferior frontal regions and its sensitivity to linear morpho-syntactic relations. Whether or not interface conditions impact the application of syntax would determine the degree of interactivity 
between subcortical and temporal structures on the one hand (responsible for narrow syntactic computations) and inferior frontal regions on the other hand (responsible for these linearity-related sub-processes). It remains purely a question for theoretical linguistics as to whether certain aspects of linearity are part of syntax proper or are instead simply 'close' to syntax (in terms of derivational stage and, presumably, processing timing) and remote from other performance systems, but we believe our proposals here can more readily situate linguistic sub-domains in a neurobiologically feasible context. The involvement of the cortico-basal ganglia-thalamo-cortical loop would be due to this complex being a major source of the brain's core timing system, with a feature of phrase structure building being rhythmicity (e.g., compare Bartolo et al. 2014 with Chomsky 2008, who both stress the importance of cyclicity).

Given the likelihood that the left inferior frontal gyrus is not in fact crucial to elementary linguistic combinatorics, recent work by Wilson et al. (2017) can be more easily integrated into this framework. These authors synthesized current research into sequence processing in primate frontal cortex and propose a ventrodorsal gradient model of frontal cortical function in sequencing operations. Reviewing comparative fMRI studies led them to propose the existence of a "conserved, bilateral, ventral frontal and opercular subsystem within frontal cortex that supports the evaluation of adjacent sequencing dependencies". The literature suggests that in both monkeys and humans, ventral regions of frontal cortex conduct processing of adjacent sequence dependencies. This leaves open the possibility that temporal regions (e.g., posterior superior temporal sulcus), alongside subcortical regions (e.g., basal ganglia, thalamus), are responsible for language-specific computational processes, as suggested here, and as we will describe in more detail in the next section. We have discussed elsewhere why we should prioritize inquiry into minimal syntactic computations to posterior temporal areas and subcortical areas over fronto-parietal areas (Murphy 2020; see also Matchin \& Hickok 2020), but for further lines of inquiry in this direction, consider the finding that chronic Broca's aphasia is associated with damage to both Broca's and Wernicke's areas (Fridriksson et al. 2015; see also Volkmer et al. 2015, 2020).

To close our discussion of the basal ganglia, we will briefly discuss this issue of the role of Broca's area in further detail. Relevant here is Lieberman's (2006) review of Broca's aphasia. Damage to Broca's area alone does not cause the full, permanent 
syndrome of Broca's aphasia, but damage deep into subcortex, including the basal ganglia, is also implicated (see Dronkers et al. 1992, D'Esposito \& Alexander 1995, Stuss \& Benson 1986). Subcortical damage with Broca's area intact can also produce speech production and language deficits (see Alexander et al. 1987, Benson \& Geschwind 1985, Mega \& Alexander 1994, Naeser et al. 1982). A similar, wellestablished and developed model of language is found in Dominey and Inui (2009). These authors suggest that cortico-striatal connections deliver a mechanism binding cortical representations of syntactic context in BA 47 to grammatical representations in BA 44. This permits the retrieval of appropriate grammatical constructions to be read-out at BA 44 via thalamo-cortical connections. The authors claim that these connections subserve forms of rule-based processing not unique to language, but which are likely recruited by the language system for regularized, overlearned grammatical computations (see also van Schouwenburg et al. 2010).

Progressing further with these concerns, Teichmann et al. (2015) discovered a "Broca-caudate pathway" which is functionally involved in natural language syntax. They found a Broca-striatum tract linking BA 45 with the left caudate head that overlapped with voxel-based lesion-symptom mapping clusters related to complex syntax. The lesion load values for this Broca-striatum tract were correlated with the complex syntax scores of their subjects. Patients with frontal/striatal damage displayed impairments in processing phrasal syntax and verbal morphosyntax, with this impairment being most clear for noncanonical sentences, though crucially independent of semantic factors pertaining to plausibility or structural factors (relative vs. non-relative). This points to issues with transformational operations of structural reorganization. These results again speak to the idea that portions of basal ganglia are implicated in externalization-related properties of syntactic information processing, and the results pertaining to phrasal syntax even indicate an earlier role in minimal phrase categorization or maintenance processes. Further, assessing the patients' forward and backward digit scans, the authors showed that there was no correlation between general working memory deficits and performance on these language tasks, pointing to a more specific syntactic deficit.

As a way of summarizing this section, we note that a necessary feature of language is that its structure-building processes (elementary syntax) need to have direct access to the categories of the items being combined and stored together as a 
larger unit. We believe it is likely that the posterior inferior frontal gyrus and the temporal lobe (being implicated in categorization processes in phonology, semantics and conceptual storage), in particular the posterior middle temporal gyrus, dynamically interact during the construction and maintenance of linguistic representations, generating the human-specific trait of phrase structure building, with this interactivity crucially modulated by subcortex. We have defended this position in detail elsewhere (Murphy 2020), where we also call upon certain measures of functional connectivity and travelling oscillatory components. For our purposes here, we focus on parallel contributions from subcortical structures and will discuss further subcortical involvement in the next section.

\section{Thalamus, hippocampus and cerebellum: Dynamic connectivity modulation, lexico-semantics and rhythmicity}

What other evidence is there for a role of subcortex in higher-order language functions? We address this question by now focussing on the thalamus, hippocampus and cerebellum.

\section{Thalamus: Lexical Memory and Global Coordination}

Beginning first with a theme introduced above concerning connectivity profiles, Schmitt et al. (2017) demonstrated that mediodorsal thalamic input can modulate prefrontal cortical connectivity, "enabling rule-specific neural sequences to emerge and thereby maintain rule representations". The authors explain that these findings expose "a previously unknown principle in neuroscience; thalamic control of functional cortical connectivity", indicating that the role of the thalamus in cognition is much wider than has typically been assumed. We have proposed elsewhere (Benítez-Burraco \& Murphy 2016, Murphy 2020) that the thalamus plays a role in lexicalizing conceptual representations through coordinating $y$-oscillating ensembles seemingly responsible for conceptual-to-lexical transformations, and we maintain that the role of this region in syntax-semantics is much more crucial than typically proposed. The thalamus is strategically placed to interface with a range of cortical regions and is impaired in cognitive disorders associated with language (Boeckx \& Benítez-Burraco 2014). It has 
been implicated more directly in lexico-semantic processing (Assaf et al. 2006). As mentioned, the cellular and synaptic mechanisms that regulate thalamic oscillations appear to have a role in controlling local and global brain rhythms (Fogerson \& Huguenard 2016, Acsády 2017). While the "outputs" of this system are detected in fronto-temporal dynamics (e.g., low frequency phase synchronization, crossfrequency coupling), it appears that subcortex forms a crucial part of the generative system which constructs, maintains in memory, and regulates linguistic structures (see Fig. 1 for a brief summary of apparent and proposed subcortical contributions to higher-order language functions).

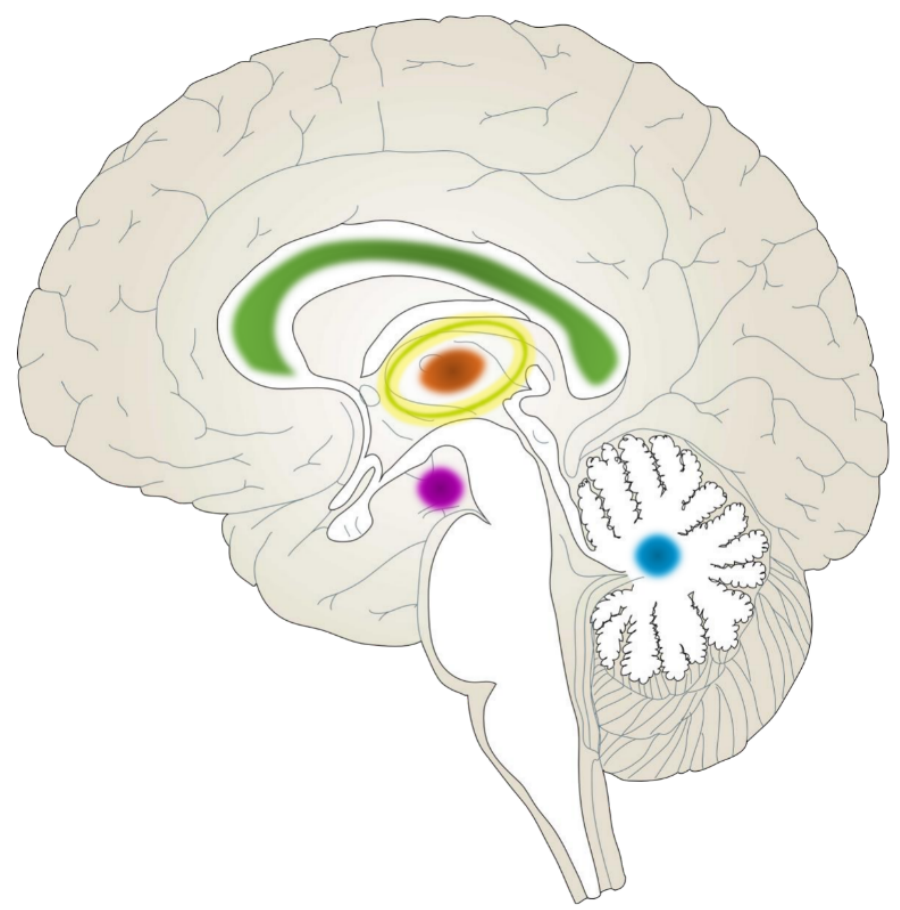

\section{BASAL GANGLIA \\ - Binary set-formation \\ - Categorized object maintenance \\ - Morphosyntactic linearization}

THALAMUS

- Cross-cortical representational integration

- Cortico-cortical connectivity modulation

- Coordination of $\gamma$-itemized lexical features

PARAHIPPOCAMPAL GYRUS

- Lexico-semantic processing

- $\theta-\gamma$ phase-amplitude coupling sequencing

- Higher-order indexing of semantic reference

\section{CEREBELLUM}

- Sequencing

- Rhythmicity

- Long-term memory

Figure 1: General schema for subcortical contributions to language. For reference, corpus callosum is marked in green.

At the representational level, cross-modular concepts widely deployed by natural language (Pietroski 2018) likely recruit thalamic nuclei such as the pulvinar and the medio-dorsal nucleus, not least because of the role of the thalamus role in modulating fronto-parietal activity, regulating cortical oscillations (Saalmann et al. 2012) and enhancing the rhythmic range of different frequency bands (Singer 2013). The anterior thalamus, for instance, is recently being considered a major site of predicting memory formation (Sweeney-Reed et al. 2015) and exhibits sensitivity to lexicality (Chrabaszcz 
et al. 2021). It is possible that this thalamic activity relates to lexical memory (see Johnson \& Knight 2015 for evidence that the thalamus plays a key role in cortical oscillations involved in memory processes). Wojtecki et al. (2017) also demonstrated a significant $\alpha$ and $\theta$ power increase and enhanced $\alpha-\theta$ coherence between the subthalamic nucleus and frontal EEG sites during a verbal generation task.

Related research points to three thalamic sub-regions responsible for coordinating oscillatory activity and facilitating memory-related processes (potentially involved in accessing lexical representations), each of which is modulated by frontal cognitive control: the medial dorsal nucleus is implicated in $\beta$ synchrony (generated by connectivity with parahippocampal/rhinal cortex), the pulvinar in a synchrony (generated by connectivity with early/visual parietal cortex), and the anterior thalamus in $\theta$ synchrony (generated by connectivity with the hippocampus; see the 'three circuit model' in Ketz et al. 2015). Thalamo-cortical interactions are increasingly being seen as central to facilitating higher cognitive processes (Pratt et al. 2017), and evidence for pulvinar expansion in humans (Pearce et al. 2013) also resonates with its presently purported role in language. Thalamic $\alpha$ has been implicated in modulating cortical $y$ power, and is also suited to synchronize distinct cortical regions oscillating at $\alpha$ (Gips et al. 2016), increasing the likelihood that it plays an important role in phrase structure building and semantic composition given the role of $\alpha$ in lexicality judgements (Strauss et al. 2015) and minimal syntactic binding (Segaert et al. 2018). Nevertheless, we stress that direct causal evidence for these dynamics with respect to language remain absent. $\beta$ coherence has also been reported between the prefrontal cortex and thalamus during working memory maintenance (Parnaudeau et al. 2013), while optogenetic suppression of the mediodorsal thalamus suppresses cortical delay activity (Schmitt et al. 2017); indicating a crucial role for thalamocortical connections in cognition.

At the level of functional connectivity, a recent model developed by Asano et al. (2021) marshals a range of evidence to suggest that the basal ganglia and thalamus exhibit functional connectivity with Broca's area to engage hierarchical control in both language and music. It appears that subcortex is involved in controlled syntactic processing, while BA 44 is independently recruited for more automatic (e.g., canonical, non-complex) syntactic processing. For instance, focal basal ganglia patients show abnormality in controlled linguistic syntax processing (Kotz et al. 2002, 2003), and a 
case report of an aphasic patient details how improved language performance posttherapy was mirrored by increased fronto-thalamic activation (Meinzer et al. 2006). Still, further empirical work is needed to explore the real-time functional connectivity profiles of subcortex and cortex during automatic and overlearned versus controlled syntactic processing, in particular given the possible sensitivity of subcortex to forms of non-complex, minimal grammatical rules. Furthermore, there is a range of evidence that seems to account for a variety of syntactic priming effects using only declarative representations (Ivanova et al. 2017, Reitter et al. 2011), explicitly avoiding procedural (and hence subcortical) ones. Further research is needed to properly tease apart the reliance of grammatical processing on particular sub-networks of the brain responsible for priming, attention and control.

Still, there seems to be little doubt that connectivity between frontal cortex and subcortex is a core regulator of cognition. Bohsali et al. (2015) document substantial structural connectivity between Broca's area and the thalamus, in particular the pulvinar. It is highly probable that the well-documented expansion of Broca's area in humans resulted in thalamic expansions, likely enhancing its ability to act as the (spatially) central oscillatory modulator in the binding of distant cortical representations. Indeed, the only sensory system which does not include a thalamic nucleus receiving sensory signals and transferring them to primary cortical regions is the olfactory system, and unless it is discovered that the sense of smell plays an unprecedentedly important role in language processing it seems safe to assume that the thalamus is central to the coordination and integration of cross-modular representations, required for typical sentence comprehension involving distinct conceptual domains. Hence, we assume a role for thalamus in aspects of lexicality, rather than specifically higher-order phrase structure inferences. Recent work also implicates the subthalamic nucleus in lexicality, with pseudoword processing resulting in greater $y$ activity in intracranial recordings than for word processing in a large cohort of Parkinson's disease patients undergoing awake deep brain stimulation (Chrabaszcz et al. 2021).

Subcortex is consequently at the centre (both spatially an operationally) of coordinating the dynamic brain activity which gives rise to the constellation of cognitive processes required for phrase structure building. Thalamic pathways contribute to oscillatory interactions between the prefrontal cortex and medial temporal lobe, two 
structures widely implicated in language comprehension. Connectivity between prefrontal cortex, the caudate nucleus and the ventral anterior nucleus in the thalamus also appears to be functionally involved in higher-order sentence processing (Jeon et al. 2014). Cortico-subcortical circuits have been implicated in a range of cognitive and motor functions (Bell \& Shine 2016). If Klostermann et al. (2013) are correct to suggest that the major thalamic functions with respect to language are "the control and adaptation of corticocortical connectivity and bandwidth for informational exchange" (a process termed "transthalamic network orchestration" by the authors), then it is likely that this region plays a crucial role in regulating other regions which are responsible for constructing and interpreting syntactic structures, through a fine-tuning of subcortical oscillations.

We have discussed elsewhere other promising directions for grounding the computational system of human language in brain dynamics, but much else remains unexplored. For example, we have here discussed some aspects of hierarchical processing and linearity, but what of the notion of unordered set-formation, which is core to models of theoretical syntax (Chomsky et al. 2019)? We have proposed that the timing of linguistic features is, broadly speaking, organized by forms of crossfrequency coupling, through which an ordered set of features can be read off from a series of fast $y$ cycles embedded inside the phase of low frequency rhythms (BenítezBurraco \& Murphy 2019). But it has recently been discovered via human intracerebral recordings that high-frequency $y$ oscillations $(70-200 \mathrm{~Hz})$ can also exhibit long-range phase synchronization, which appears behaviorally-relevant in a response-inhibition task (Arnulfo et al. 2020). This broadens the scope for simultaneously active (bound) linguistic features to be organized into a unit of cross-cortical manipulation and computation (likely coordinated via low frequency activity in the service of workspace construction; Murphy 2020), approximating much closer the formal notion of unordered binary set-formation (Chomsky et al. 2019) than the linear feature-clocking of phaseamplitude coupling suggested in our previous work. The joint coordination and activation of cross-cortical linguistic features (free from any serialization via phaseamplitude coupling) may constitute one of the earliest stages of phrase structure building, with these coordinated high-frequency $y$ cycles being then phase-coupled to the familiar lower frequency bands seemingly responsible for generating structural 
inferences. Again, we point to the thalamus as a strong candidate for modulating and constraining cross-cortical activity, synchronizing distant cortical areas.

\section{Hippocampus: Representational Binding}

Moving to other subcortical regions, recent work has shown that left hippocampus is involved in hierarchical linguistic interpretation (Blank et al. 2016), linguistic semantics and referential processing (Piai et al. 2016) and auditory language comprehension (Wilson et al. 2008). Vanier et al. (2019) discovered that, relative to apes and monkeys, the human hippocampal formation shows an increase in size in the CA3, subiculum and rhinal cortex regions, and also an increase in the neocortex; expansions which might underlie our unique episodic memory capacity, as the authors speculate, but also our unique ability to string together large clusters of cortical linguistic features into coherent lexical items and phrases (for landmark work in this domain, see Piai et al. 2016; see also Benítez-Burraco 2021). The hippocampus also seems particularly suited to facilitate cross-modular interactions, since it has been argued to play a role in integrating 'what' and 'where' information in the perirhinal cortex through the lateral entorhinal cortex and the postrhinal cortex through the medial entorhinal cortex (Fernández-Ruiz \& Oliva 2016). Henin et al. (2021), using intracranial recordings in humans, exposed patients to auditory and visual sequences containing temporal regularities. Early on, both lower-level features, such as syllables, and learned units, such as words, were tracked neurally, while in later periods of the experiment only learned units were tracked. In particular, associative regions and hippocampus encoded the ordinal position and identity of hierarchical units.

Moreover, as Covington and Duff (2016) review, it is increasingly being shown that "the same hippocampal computations used in support of memory are also used for language processing", pointing to a shared neural code for particular computations. $\theta-\gamma$ phase-phase coupling in the human hippocampus has also been shown to be involved in multi-item working memory maintenance (Chaieb et al. 2015), and we have previously argued that different cognitive domains may construct representational stacks via different types of couplings (Murphy 2020). In essence, it is possible that some domain-general stack-construction algorithms implemented via forms of oscillatory coupling are exploited by certain sub-components of the language system 
(Benítez-Burraco \& Murphy 2019), from sub-lexical feature concatenation to more abstract representations denoting aspects of linguistically-constructed situation models (Horner et al. 2015). Again speaking to the sub-lexical level, the hippocampus, globus pallidus and caudate nucleus have been recently implicated in orthographic single-word processing (Braun et al. 2019), seemingly being involved in certain aspects of mapping orthographic forms onto long-term memory representations, potentially via basal ganglia maintaining orthographic forms in working memory and hippocampal activity indexing the mapping to stored representations.

Nevertheless, the role of hippocampus appears to be somewhat specific: Binder et al. (2020) found that in epilepsy patients picture naming performance decline was unrelated to hippocampal resections, but was rather linked to fusiform gyrus resections (see also Forseth et al. 2018). Hippocampus may be recruited for specific language functions pertaining to representational binding, but seems not to be causally implicated in visual object naming abilities. The possiblity of post-resection reorganization is a possibility here, and indeed pre-resection reorganization, given that the presence of epileptic tissue may trigger reorganization years before surgery, possibly to contralateral hippocampal sites (Trimmel et al. 2019; see also TrebuchonDa Fonseca et al. 2009 for discussion of how temporal lobe epilepsy may induce functional deafferentation between non-local structures).

While the causal role of hippocampus in language seems unclear (and also the parahippocampal gyrus, which seems implicated in episodic memory and context processing; Aminoff et al. 2013), the real-time dynamical engagement of this region has been explored extensively. Reviewing experimental literature investigating rodents and humans, Hanslmayr et al. (2016) propose a general trend: While hippocampal $\theta-y$ phase-amplitude coupling mediates the binding of distinct episodic memory representations, the desynchronization of slower neocortical rhythms ( $\alpha$ and $\beta$ ) also appears to mediate the encoding of episodic memories; exposing the inhibitory role of $\alpha$ in aiding successful memory encoding and retrieval by other brain regions. The hippocampal synchronisation system therefore appears to bind information, while the neocortical desynchronisation system stores the representational content (see also Arnulfo et al. 2020). Both episodic memory and language involve the binding of discontiguous representations such as distinct memories and unrelated semantic features. This crucial computational similarity may imply that both systems recruit the 
same hippocampal-neocortical system for aspects of their representational triggering and storage (again, see Piai et al. 2016 for hippocampal theta dynamics in natural language lexico-semantics). To emphasize a point already made: Certain frontotemporal cortical clusters may be sensitive to the output of natural language syntactic computation (and can hence be readily detected via functional neuroimaging), but the early stages of initial generation are likely reliant on subcortex to a much greater degree than typically acknowledged.

The role of hippocampus in language may go even beyond this. Recent work (Ellamil et al. 2016) has demonstrated the central role of the hippocampus in spontaneous thought generation (or 'mind-wandering', which seemingly occupies 20$50 \%$ of daily life; Killingsworth \& Gilbert 2010), with a large portion of cells in this region being involved in short-distance and long-distance connections. This may contribute to an explanation for the existence of some of the qualities of spontaneous thought, such as the fact that it often contains semantic representations "of wild diversity and content" (Ellamil et al. 2016), since hippocampal long-range connections interface with numerous cortical regions which store a variety of representations. Likewise, much of sponataneous linguistic thought crosses modular boundaries. The creative expression of cross-modular concepts is paramount to ordinary linguistic productivity, in both interpretation and production, and these hippocampal dynamics appear to be part of the basis for such behavior. As such, the hippocampus might not only explain features of "the restless nature of our minds" (Ellamil et al. 2016: 195), but could also more specifically shed light on lexico-semantics (relatedly, Mišić et al. 2014 provide evidence that the hippocampus is a crucial convergence zone for information flow).

\section{Cerebellum: The Syntactic Time Keeper}

Lastly, there are recent indications that the cerebellum may play a role in certain aspects of cognition. Portions of cerebellum (lobule VIIb/VIIla) hold objects from visual working memory (Brissenden et al. 2021), crucial for semantic categorization. As we have previously reviewed (Murphy 2019), the cerebellum also plays a role in long-term memory, while Nozaradan et al. (2017) reveal how the cerebellum and basal ganglia are involved in the neural representations of rhythmic sequences. Cerebellar lesions can lead to non-motor language deficits impacting syntax, semantics and sentence 
formation (for review, see Moberget \& Ivry 2019), with the cerebellum having been implicated in linguistic syntax (Adamaszek \& Kirkby 2016), in the maintenance of phrase structure in working memory (Mariën et al. 2001), and patients with cerebellar lesions have displayed reduced abilities to discriminate between grammatical and ungrammatical sentences (Justus 2004). Cerebellar contributions to language appear to be structured by major pathways comprising the cerebrocortico-pontocerebellocortico-dentato-thalamocerebrocortical and cerebrocortico-rubro-olivoneodentato-cerebrocortical loops (Adamaszek \& Kirkby 2016, Schmahmann \& Pandya 1997). Typically, right-hemispheric cerebellum activity is coordinated with dominant left-hemispheric activity, forming part of the extended language network (Justus 2014), although ipsilateral activity has also been documented (Adamaszek \& Kirkby 2016). Right and left cerebellum, in isolation but also together, have been claimed to be essential for linguistic tasks, although a common generalization is that right cerebellum responds greater for high-frequency information and language, while left cerebellum responds greater for low-frequency information and singing (Callan et al. 2007).

Cerebellar interfacing via cerebrocerebellar pathways with the left temporal and prefrontal gyrus also appears involved in language perception and production, such as in verbal fluency, word stem completion, word and letter generation, and semantic processing (Murdoch 2010). Along with the pre-SMA, SMA and basal ganglia, the cerebellum has also been implicated in the coordination of temporal structure (Kotz \& Schwartze 2010). Based on event-related potential responses to morphosyntactically correct versus incorrect sentence processing, other authors suggest that the cerebellum offers "a preattentive support role", being involved in "forwarding results of syntactic analysis to frontotemporal pathways" via the thalamus (Adamaszek \& Kirkby 2016: 89). The cerebellum appears to regulate the temporal integration of items in working memory relevant to morphosyntactic information, handling the temporal coordination of grammatically-relevant memory items. Cerebellum-related deficits are often performance-related aspects of language (e.g., monitoring and timing of sequences, and the detection of violations of predicted grammatical rules; Booth et al. 2007, Mariën \& Borgatti 2018), though given the small number of direct recordings of the human cerebellum during tasks manipulating some component of syntaxsemantics, it also seems too early to make strong conclusions here. 


\section{Conclusion}

The potential for empirical assessments of the architectural claims we have made here is rapidly increasing. For instance, intracranial electrocorticographic research during deep brain stimulation surgery has recently been shown to not significantly alter complication rates (Sisterson et al. 2021), suggesting that academic research into the assessment of basal ganglia-thalamocortical circuit physiology is a safe procedure, opening up new avenues for investigating subcortical involvement in natural language syntax-semantics.

Overall, since subcortex has readily been acknowledged to be essential for a range of higher cognitive functions, it seems reasonable to expect important contributions from these regions with respect to language processing. The small number of subcortical structures we have reviewed here are something of a motley crew, each contributing quite distinct computational roles and exhibiting widely different connectivity profiles, and we expect future research will expand the list of subcortical structures known to be recruited for language. In brief, we have here tried to marshall a range of evidence suggesting that subcortex does in fact play an essential role in natural language syntax-semantics - a suggestion which, if further validated in future work, would demand a considerable revision to neurolinguistic models. 


\section{References}

Ackermann, H., \& Ziegler, W. (2013). A "birdsong perspective" on human speech production. In Bolhuis, J.J., \& Everaert, M. (Eds). Birdsong, Speech, and Language: Exploring the Evolution of Mind and Brain. Cambridge, MA: MIT Press. 331-352.

Acsády, L. (2017). The thalamic paradox. Nature Neuroscience 20: 901-902.

Adamaszek, M., \& Kirkby, K.C. (2016). Cerebellum and grammar processing. In P. Mariën \& M. Manto (Eds.), The Linguistic Cerebellum (pp. 81-105). Amsterdam: Elsevier.

Alexander, M.P., Naeser, M.A., \& Palumbo, C.L. (1987). Correlations of subcortical CT lesion sites and aphasia profiles. Brain 110: 961-991.

Aminoff, E.M., Kveraga, K., \& Bar, M. (2013). The role of the parahippocampal cortex in cognition. Trends in Cognitive Sciences 17(8): 379-390.

Arnulfo, G., Wang, S.H., Myrov, V., Toselli, B., Hirvonen, J., Fato, M.M., Nobili, L., Cardinale, F., Rubino, A., Zhigalov, A., Palva, S., \& Palva, J.M. (2020). Long-range phase synchronization of high-frequency oscillations in human cortex. Nature Communications 11: 5363. 
Asano, R., Boeckx, C., \& Seifert, U. (2021). Hierarchical control as a shared neurocognitive mechanism for language and music. Cognition 216: 104847.

Assaf, M., Calhoun, V.D., Kuzu, C.H., Kraut, M.A., Rivkin, P.R., Hart, J. Jr., Pearlson, G.D. (2006). Neural correlates of the object-recall process in semantic memory. Psychiatry Research 147: 115-126.

Balari, S., \& Lorenzo, G. (2013). Computational Phenotypes: Towards an Evolutionary Developmental Biolinguistics. Oxford: Oxford University Press.

Bartolo, R., Prado, L., \& Merchant, H. (2014). Information processing in the primate basal ganglia during sensory-guided and internally driven rhythmic tapping. Journal of Neuroscience 34: 3910-3923.

Bell, P.T., \& Shine, J.M. (2016). Subcortical contributions to large-scale network communication. Neuroscience and Biobehavioral Reviews 71: 313-322.

Benítez-Burraco, A. (2021). Mental time travel, language evolution, and human selfdomestication. Cognitive Processing https://doi.org/10.1007/s10339-020-01005-2.

Benítez-Burraco, A., Di Pietro, L., Barba, M., \& Lattanzi, W. (2017). Schizophrenia and human self-domestication: an evolutionary linguistics approach. Brain, Behavior and Evolution 89: 162-184.

Benítez-Burraco, A., \& Murphy, E. (2016). The oscillopathic nature of language deficits in autism: from genes to language evolution. Frontiers in Human Neuroscience 10: 120.

Benítez-Burraco, A., \& Murphy, E. (2019). Why brain oscillations are improving our understanding of language. Frontiers in Behavioral Neuroscience 13: 190.

Benítez-Burraco, A., Theofanopoulou, C., \& Boeckx, C. (2018). Globularization and domestication. Topoi 37: 265-278.

Benson, D.F., \& Geschwind, N. (1985). Aphasia and related disorders: a clinical approach. Mesulam, M.M. (Ed.). Principles of Behavioral Neurology. Philadelphia: F.A. Davis. 193-228.

Bice, K., Yamasaki, B.L., \& Prat, C.S. (2020). Bilingual language experience shapes resting-state brain rhythms. Neurobiology of Language 1(3): 288-318. 
Binder, J.R., Tong, J-Q., Pillay, S.B., Conant, L.L., Humphries, C.J., Raghavan, M., Mueller, W.M., Busch, R.M., Allen, L., Gross, W.L. et al. (2020). Temporal lobe regions essential for preserved picture naming after left temporal epilepsy surgery. Epilepsia 61: 1939-1948.

Blank, I., Duff, M. C., Brown-Schmidt, S., \& Fedorenko, E. (2016). Expanding the language network: domain-specific hippocampal recruitment during high-level linguistic processing. bioRxiv doi.org/10.1101/091900.

Boeckx, C., \& Benítez-Burraco, A. (2014). The shape of the human language-ready brain. Frontiers in Psychology 5: 282.

Bohsali, A.A., Triplett, W., Sudhyadhom, A., Gullett, J.M., McGregor, K., FitzGerald, D.B., Mareci, T., White, K., \& Crosson, B. (2015). Broca's area - thalamic connectivity. Brain and Language 141: 80-88.

Booth, J.R., Wood, L., Lu, D., Houk, J.C., \& Bitan, T. (2007). The role of the basal ganglia and cerebellum in language processing. Brain Research 1133: 136-144.

Braun, M., Kronbichler, M., Richlan, F., Hawelka, S., Hutzler, F., \& Jacobs, A.M. (2019). A model-guided dissociation between subcortical and cortical contributions to word recognition. Scientific Reports 9: 4506.

Brissenden, J.A., Tobyne, S.M., Halko, M.A., \& Somers, D.C. (2021). Stimulus-specific visual working memory representations in human cerebellar lobule VIIb/VIIla. Journal of Neuroscience 41(5): 1033-1045.

Bruner, E., \& Gleeson, B.T. (2019). Body cognition and self-domestication in human evolution. Frontiers in Psychology 10: 1111.

Burgaleta, M., Sanjuán, A., Ventura-Campos, N., Sebastian-Galles, N., \& Ávila, C. (2016). Bilingualism at the core of the brain. Structural differences between bilinguals and monolinguals revealed by subcortical shape analysis. Neurolmage 125: 437-445.

Burroughs, A., Kazanina, N., \& Burroughs, C. (2021). Grammatical category and the neural processing of phrases. Scientific Reports 11: 2446.

Buzsáki, G., \& Llinás, R. (2017). Space and time in the brain. Science 358: 482-485. 
Callan, D.E., Kawato, M., Parsons, L., \& Turner, L. (2007). Speech and song: the role of the cerebellum. Cerebellum 8: 1-7.

Cargnelutti, E., Tomasino, B., \& Fabbro, F. (2019). Language brain representation in bilinguals with different age of appropriation and proficiency of the second language: a meta-analysis of functional imaging studies. Frontiers in Human Neuroscience 13: 154.

Chaieb, L., Leszczynski, M., Axmacher, N., Höhne, M., Elger, C. E., \& Fell, J. (2015). Theta-gamma phase-phase coupling during working memory maintenance in the human hippocampus. Cognitive Neuroscience 6: 149-157.

Chomsky, N. (2008). On phases. Freidin, R., Otero, C. P., \& Zubizarreta, M. L. (Eds). Foundational Issues in Linguistic Theory: Essays in Honor of Jean-Roger Vergnaud. Cambridge, MA: MIT Press. 133-166.

Chomsky, N. (2020). Minimalism: Where we are now, and where we are going. Lecture at the $161^{\text {st }}$ Meeting of the Linguistic Society of Japan.

Chomsky, N., Gallego, Á.J., \& Ott, D. (2019). Generative grammar and the faculty of language: insights, questions, and challenges. Catalan Journal of Linguistics Special Issue 229-261.

Chrabaszcz, A., Wang, D., Lipski, W.J., Crammond, D.J., Shaiman, S., Dickey, M.W., Holt, L.L., Turner, R.S., Fiez, J.A., \& Richardson, R.M. (2021). Simultaneously recorded subthalamic and cortical LFPs reveal different lexicality effects during reading aloud. Journal of Neurolinguistics 60: 101019.

Corballis, M.C. (2002). From Hand to Mouth: The Origins of Language. Princeton, NJ: Princeton University Press.

Corina, D.P., Gibson, E.K., Martin, R., Poliakov, A., Brinkley, J., Ojemann, G.A. (2005). Dissociation of action and object naming: evidence from cortical stimulation mapping. Human Brain Mapping 24(1): 1-10.

Covington, N.V., \& Duff, M.C. (2016). Expanding the language network: direct contributions from the hippocampus. Trends in Cognitive Sciences 20(12): 869-870. 
Crinion, J., Holland, A.L., Copland, D.A., Thompson, C.K., \& Hillis, A.E. (2013). Neuroimaging in aphasia treatment research: quantifying brain lesions after stroke. Neurolmage 73: 208-214.

Dean, H.L., Hagan, M.A., \& Pesaran, B. (2012). Only coherent spiking in posterior parietal cortex coordinates looking and reaching. Neuron 73(4): 829-841.

Deco, G., Cabral, J., Woolrich, M.W., Stevner, A.B.A., van Hartevelt, T.J., \& Kringelbach, M. L. (2017). Single or multi-frequency generators in on-going brain activity: a mechanistic whole-brain model of empirical MEG data. Neurolmage 152: 538-550.

Denenberg, V.H. (1981). Hemispheric laterality in animals and the effects of early experience. Behavioral and Brain Sciences 4: 1-49.

D’Esposito, M. \& Alexander, M.P. (1995). Subcortical aphasia: distinct profiles following left putaminal hemorrhage. Neurology 45: 38-41.

Dominey, P., \& Inui, T. (2009). Cortico-striatal function in sentence comprehension: insights from neurophysiology and modeling. Cortex 45(8): 1012-1018.

Dronkers, N.F., Shapiro, J.K., Redfern, B., \& Knight, R.T. (1992). The role of Broca's area in Broca's aphasia. Journal of Clinical and Experimental Neuropsychology 14: 52-53.

Duffy, J.R. (2005). Motor Speech Disorders: Substrates, Differential Diagnosis, and Management. 2nd ed. St. Louis, MO: Elsevier Mosby.

Ellamil, M., Fox, K.C., Dixon, M.L., Pritchard, S., Todd, R.M., Thompson, E., \& Christoff, K. (2016). Dynamics of neural recruitment surrounding the spontaneous arising of thoughts in experienced mindfulness practitioners. Neurolmage 136: 186196.

Engel, A.K. \& Fries, P. (2010). Beta-band oscillations - signalling the status quo? Current Opinion in Neurobiology 20(2): 156-165.

Fernández-Ruiz, A., \& Oliva, A. (2016). Distributed representation of 'what' and 'where' information in the parahippocampal region. Journal of Neuroscience 36(32): 8286-8288. 
Flick, G., Oseki, Y., Kaczmarek, A.R., Al Kaabi, M., Marantz, A., \& Pylkkänen, L. (2018). Building words and phrases in the left temporal lobe. Cortex 106: 213-236.

Flick, G., \& Pylkkänen, L. (2020). Isolating syntax in natural language: MEG evidence for an early contribution of left posterior temporal cortex. Cortex 127: 42-57.

Fogerson, P.M., \& Huguemard, J.R. (2016). Tapping the breaks: cellular and synaptic mechanisms that regulate thalamic oscillations. Neuron 92: 687-704.

Forseth, K.J., Kadipasaoglu, C.M., Conner, C.R., Hickok, G., Knight, R.T., \& Tandon, N. (2018). A lexical semantic hub for heteromodal naming in middle fusiform gyrus. Brain 141: 2112-2126.

Fridriksson, J., Fillmore, P., Guo, D., \& Rorden, C. (2015). Chronic Broca's aphasia is caused by damage to Broca's and Wernicke's areas. Cerebral Cortex 25(12): 46894696.

Friederici, A.D. (2017). Language in Our Brain. Cambridge, MA: MIT Press.

Friederici, A.D., Bahlmann, J., Heim, S., Schubotz, R.I., \& Anwander, A. (2006). The brain differentiates human and non-human grammars: functional localization and structural connectivity. PNAS 103: 2458-63.

Friederici, A.D., Rüschemeyer, S-A., Hahne, A., \& Fiebach, C.J. (2003). The role of left inferior frontal and superior temporal cortex in sentence comprehension: localizing syntactic and semantic processes. Cerebral Cortex 13(2): 170-177.

Gabi, M., Neves, K., Masseron, C., Ribeiro, P.F.M., Ventura-Antunes, L., Torres, L., Mota, B., \& Herculano-Houzel, S. (2016). No relative expansion of the number of prefrontal neurons in primate and human evolution. PNAS 113(34): 9617-9622.

Gehrig, J., Michalareas, G., Forster, M-T., Lei, J., Hok, P., Laufs, H., Senft, C., Seifert, V., Schoffelen, J-M., Hanslmayr, S., \& Kell, C.A. (2019). Low frequency oscillations code speech during verbal working memory. Journal of Neuroscience 39(33): 64986512.

Gibson, K.R., \& Jessee, S. (1999). Language evolution and expansions of multiple neural processing areas. In B. King (Ed.) The Evolution of Language: Assessing the Evidence from the Non-Human Primates. Santa Fe, NM: School for American Research. 189-228. 
Gips, B., van der Eerden, J.P.J.M., \& Jensen, O. (2016). A biologically plausible mechanism for neuronal coding organized by the phase of alpha oscillations. European Journal of Neuroscience 44(4): 2147-2161.

Gollo, L.L., Roberts, J.A., \& Cocchi, L. (2017). Mapping how local perturbations influence systems-level brain dynamics. Neurolmage 160: 97-112.

Griffiths, J.D., Marslen-Wilson, W.D., Stamatakis, E.A., \& Tyler, L.K. (2013). Functional organization of the neural language system: Dorsal and ventral pathways are critical for syntax. Cerebral Cortex 23: 139-147.

Haber, S.N., \& Calzavara, R. (2009). The cortico-basal ganglia integrative network: the role of the thalamus. Brain Research Bulletin 78: 69-74.

Halassa, M.M., Chen, Z., Wimmer, R.D., Brunetti, P.M., Zhao, S., Zikopoulos, B., Wang, F., Brown, E.N., \& Wilson, M.A. (2014). State-dependent architecture of thalamic reticular subnetworks. Cell 158(4): 808-821.

HansImayr, S., Staresina, B.P., \& Bowman, H. (2016). Oscillations and episodic memory: addressing the synchronization/desynchronization conundrum. Trends in Neurosciences 39(1): 16-25.

Haynes, W.I.A., \& Haber, S.N. (2013). The organization of prefrontal-subthalamic inputs in primates provides an anatomical substrate for both functional specificity and integration: implications for basal ganglia models and deep brain stimulation. Journal of Neuroscience 33(11): 4804-4814.

Henin, S., Turk-Browne, N.B., Friedman, D., Liu, A., Dugan, P., Flinker, A., Doyle, W., Devinsky, O., \& Melloni, L. (2021). Learning hierarchical sequence representations across human cortex and hippocampus. Science Advances 7(8): eabc4530.

Hernandez, A.E., \& Li, P. (2007). Age of acquisition: its neural and computational mechanisms. Psychological Bulletin 133(4): 638-650.

Hervais-Adelman, A., Egorova, N., \& Golestani, N. (2018). Beyond bilingualism: multilingual experience correlates with caudate volume. Brain Structure and Function 223: 3495-3502. 
Horner, A.J., Bisby, J.A., Bush, D., Wen-Jing, L., \& Burgess, N. (2015). Evidence for holistic episodic recollection via hippocampal pattern completion. Nature Communications 6: 7462.

Hornstein, N. (2009). A Theory of Syntax: Minimal Operations and Universal Grammar. Cambridge: Cambridge University Press.

Hornstein, N., \& Pietroski, P. (2009). Basic operations: minimal syntax-semantics. Catalan Journal of Linguistics 8: 113-139.

Hoshi, K. (2017). Lenneberg's contributions to the biology of language and child aphasiology: resonation and brain rhythmicity as key mechanisms. Biolinguistics 11(SI): 83-113.

Hoshi, K. (2018). Merge and labeling as descent with modification of categorization: a neo-Lennebergian approach. Biolinguistics 12: 39-54.

Hoshi, K. (2019). More on the relations among categorization, Merge and labeling, and their nature. Biolinguistics 13: 1-21.

Hoshi, K., \& Miyazato, K. (2016). Architecture of human language from the perspective of a case of childhood aphasia-Landau-Kleffner syndrome. Biolinguistics 10: 136196.

Huettel, S.A., Mack, P.B., \& McCarthy, G. (2002). Perceiving patterns in random series: Dynamic processing of sequence in prefrontal cortex. Nature Neuroscience 5: 485-490.

Ivanova, I., Branigan, H.P., McLean, J.F., Costa, A., \& Pickering, M.J. (2017). Do you what I say? People reconstruct the syntax of anomalous sentences. Language, Cognition and Neuroscience 32(2): 175-189.

Jeon, H-A., Anwander, A., \& Friederici, A.D. (2014). Functional network mirrored in the prefrontal cortex, caudate nucleus, and thalamus: high-resolution functional imaging and structural connectivity. Journal of Neuroscience 34(28): 9202-9212.

Jiménez-Bravo, M., Marrero, V., \& Benítez-Burraco, A. (2017). An oscillopathic approach to developmental dyslexia: from genes to speech processing. Behavioural Brain Research 329: 84-95. 
Johari, K., Walenski, M., Reifegerste, J., Ashrafi, F., Behroozmand, R., Daemi, M., \& Ullman, M.T. (2019). A dissociation between syntactic and lexical processing in Parkinson's disease. Journal of Neurolinguistics 51: 221-253.

Johnson, E. J., \& Knight, R. T. (2015). Intracranial recordings and human memory. Current Opinion in Neurobiology 31: 18-25.

Justus, T. (2004). The cerebellum and english grammatical morphology: evidence from production, comprehension, and grammaticality judgements. Journal of Cognitive Neuroscience 16(7): 1115-1130.

Kepinska, O., de Rover, M., Caspers, J., \& Schiller, N.O. (2018). Connectivity of the hippocampus and Broca's area during acquisition of a novel grammar. Neurolmage 165: 1-10.

Ketz, N.A., Jensen, O., \& O'Reilly, R.C. (2015). Thalamic pathways underlying prefrontal cortex-medial temporal lobe oscillatory interactions. Trends in Neurosciences 38(1): 3-12.

Killingsworth, M.A., \& Gilbert, D.T. (2010). A wandering mind is an unhappy mind. Science 330: 932.

Klostermann, F., Krugel, L.K., \& Ehlen, F. (2013). Functional roles of the thalamus for language capacities. Frontiers in Systems Neuroscience 7: 32.

Kol, A., Adamsky, A., Groysman, M., Kreisel, T., London, M., \& Goshen, I. (2020). Astrocytes contribute to remote memory formation by modulating hippocampalcortical communication during learning. Nature Neuroscience 23: 1229-1239.

Kopell, N.J., Kramer, M.A., Malerba, P., \& Whittington, M.A. (2010). Are different rhythms good for different functions? Frontiers in Human Neuroscience 4: 187.

Kotz, S.A., Frisch, S., Von Cramon, D.Y., \& Friederici, A.D. (2003). Syntactic language processing: ERP lesion data on the role of the basal ganglia. Journal of the International Neuropsychological Society 9(7): 1053-1060.

Kotz, S.A., Frisch, S., Werheid, K., Hein, G., von Cramon, D.Y., \& Friederici, A.D. (2002). The role of the basal ganglia in syntactic language processing: Event-related potential evidence from different patient populations and syntactic paradigms. Brain and Language 83(1): 68-70. 
Kotz, S.A., \& Schwartze, M. (2010). Cortical speech processing unplugged: a timely subcortico-cortical framework. Trends in Cognitive Sciences 14(9): 393-399.

Koziol, L.F., Budding, D.E., \& Suth, A. (2009). Subcortical Structures and Cognition: Implications for Neuropsychological Assessment. New York: Springer.

Lenneberg, E.H. (1967). Biological Foundations of Language. New York: John Wiley and Sons.

Li, Q., Del Ferraro, G., Pasquini, L., Peck, K.K., Makse, H.A., \& Holodny, A.I. (2020). Core language brain networks for fMRI language task used in clinical application. Network Neuroscience 4(1): 134-154.

Lieberman, P. (2006). Toward an Evolutionary Biology of Language. Cambridge, MA: Harvard University Press.

Lieberman, P. (2015). Language did not spring forth 100,000 years ago. PLoS Biology 13(2): e1002064.

Lipski, W.J., Alhourani, A., Pirnia, T., Jones, P.W., Dastolfo-Hromack, C., Helou, L.B., Crammond, D.J., Shaiman, S., Dickey, M.W., Holt, L.L., Turner, R.S., Fiez, J.A., \& Richardson, R.M. (2018). Subthalamic nucleus neurons differentially encode early and late aspects of speech production. Journal of Neuroscience 38(24): 5620-5631.

Mariën, P., \& Borgatti, R. (2018). Language and the cerebellum. Handbook of Clinical Neurology 154: 181-202.

Mariën, P., Engelborghs, S., Fabbro, F., \& De Deyn, P. P. (2001). The lateralized linguistic cerebellum: a review and a new hypothesis. Brain and Language 79(3): 580600.

Mareschal, D., Quinn, P.C., \& Lea, S.E.G. (Eds.). (2010). The Making of Human Concepts. Oxford: Oxford University Press.

Martin, C. (2016). The cryptic cortex. Current Biology 26: R941-R945.

Martin, C., \& Ravel, N. (2014). Beta and gamma oscillatory activities associated with olfactory memory tasks: different rhythms for different functional networks? Frontiers in Behavioral Neuroscience 8: 218. 
Martins, M.D., \& Villringer, A. (2018). The human arcuate fasciculus provides specific advantages to process complex sequential stimuli, not hierarchies in general. Cuskley, C., Flaherty, M., McCrohon, L., Little, H., Ravignani, A., \& Verhoef, T. (Eds). The Evolution of Language: Proceedings of the 12th International Conference (Evolang 12). Torun: Nicolaus Copernicus University. 287-289.

Matchin, W., Hammerly, C., \& Lau, E. (2017). The role of the IFG and pSTS in syntactic prediction: Evidence from a parametric study of hierarchical structure in fMRI. Cortex 88: 106-123.

Matchin, W., \& Hickok, G. (2020). The cortical organization of syntax. Cerebral Cortex 30(3): 1481-1498.

Mathieu, E. (2016). The wh-parameter and radical externalization. Eguren, L., Fernández-Soriano, O., \& Mendikoetxea, A. (Eds.). Rethinking Parameters. Oxford: Oxford University Press. 252-290.

Mayberry, R.I., Davenport, T., Roth, A., \& Halgren, E. (2018). Neurolinguistic processing when the brain matures without language. Cortex 99: 390-403.

Mega, M.S., \& Alexander, M.P. (1994). Subcortical aphasia: the core profile of capsulostriatal infarction. Neurology 44: 1824-1829.

Meinzer, M., Flaisch, T., Obleser, J., Assadollahi, R., Djundja, D., Barthel, G., \& Rockstroh, B. (2006). Brain regions essential for improved lexical access in an aged aphasic patient: a case report. BMC Neurology 6: 28.

Meyer, L., Sun, Y., \& Martin, A.E. (2020). Synchronous, but not entrained: exogenous and endogenous cortical rhythms of speech and language processing. Language, Cognition and Neuroscience 35(9): 1089-1099.

Mišić, B., Goni, J., Betzel, R.F., Sporns, O., \& Mclntosh, A.R. (2014). A network convergence zone in the hippocampus. PLoS Computational Biology 10: e1003982.

Moberget, T., \& Ivry, R.B. (2019). Prediction, psychosis, and the cerebellum. Biological Psychiatry: Cognitive Neuroscience and Neuroimaging 4(9): 820-831.

Monchi, O., Petrides, P., Petre, V., Worsley, K., \& Dagher, A. (2001). Wisconsin card sorting revisited: Distinct neural circuits participating in different stages of the task 
identified by event-related functional magnetic resonance imaging. Journal of Neuroscience 21: 7733-7741.

Moreno, A., Limousin, F., Dehaene, S., \& Pallier, C. (2018). Brain correlates of constituent structure in sign language comprehension. Neurolmage 167: 151-161.

Moro, A., Tettamanti, M., Perani, D., Donati, C., Cappa, S.F., \& Fazio, F. (2001). Syntax and the brain: disentangling grammar by selective anomalies. Neurolmage 13: 110-118.

Murdoch, B. E. (2010). The cerebellum and language: historical perspective and review. Cortex 46: 858-868.

Murphy, E. (2015). The brain dynamics of linguistic computation. Frontiers in Psychology 6: 1515.

Murphy, E. (2016). Phasal eliminativism, anti-lexicalism, and the status of the unarticulated. Biolinguistics 10: 21-50.

Murphy, E. (2018). Interfaces (travelling oscillations) + recursion (delta-theta code) = language. Luef, E., \& Manuela, M. (Eds). The Talking Species: Perspectives on the Evolutionary, Neuronal and Cultural Foundations of Language. Graz: Unipress Graz Verlag. 251-269.

Murphy, E. (2019). No country for Oldowan men: emerging factors in language evolution. Frontiers in Psychology 10: 1448.

Murphy, E. (2020). The Oscillatory Nature of Language. Cambridge: Cambridge University Press.

Murphy, E., \& Benítez-Burraco, A. (2016). Bridging the gap between genes and language deficits in schizophrenia: an oscillopathic approach. Frontiers in Human Neuroscience 10: 422.

Murphy, E., \& Benítez-Burraco, A. (2017). Language deficits in schizophrenia and autism as related oscillatory connectomopathies: an evolutionary account. Neuroscience and Biobehavioral Reviews 83: 742-764.

Murphy, E., \& Benítez-Burraco, A. (2018). Toward the language oscillogenome. Frontiers in Psychology 9: 1999. 
Murugesan, B.G., Jafroodifar, A., Anilkumar, A.C., \& Leontieva, L. (2020). Differential diagnosis of Landau-Kleffner syndrome versus post encephalitis syndrome in a 13year-old boy with autism spectrum disorder. Cureus 12(7): e9385.

Naeser, M.A., Alexander, M.P., Helms-Estabrooks, N., Levine, H.L., Laughlin, S.A., \& Geschwind, N. (1982). Aphasia with predominantly subcortical lesion sites: Descriptions of three capsular/putaminal aphasia syndromes. Archives of Neurology 39: 2-14.

Nozaradan, S., Schwartze, M., Obermeier, C., \& Kotz, S.A. (2017). Specific contributions of basal ganglia and cerebellum to the neural tracking of rhythm. Cortex 95: 156-168.

Palomero-Gallagher, N., \& Zilles, K. (2019). Differences in cytoarchitecture of Broca's region between human, ape and macaque brains. Cortex 118: 132-153.

Parnaudeau, S., O'Neill, P.K., Bolkan, S.S., Ward, R.D., Abbas, A.I., Roth, B.L., Balsam, P.D., Gordon, J.A. \& Kellendonk, C. (2013). Inhibition of mediodorsal thalamus disrupts thalamofrontal connectivity and cognition. Neuron 77: 1151-1162.

Parvizi, J. (2009). Corticocentric myopia: old bias in new cognitive sciences. Trends in Cognitive Sciences 13(8): 354-359.

Pearce, E., Stringer, C., \& Dunbar, R. I. M. (2013). New insights into differences in brain organization between Neanderthals and anatomically modern humans. Proceedings of the Royal Society B 280: 20130168.

Petersson, K. M., Folia, V., \& Hagoort, P. (2012). What artificial grammar learning reveals about the neurobiology of syntax. Brain \& Language 120(2): 83-95.

Piai, V., Anderson, K.L., Lin, J.J., Dewar, C., Parvizi, J., Dronkers, N.F., \& Knight, R.T. (2016). Direct brain recordings reveal hippocampal rhythm underpinnings of language processing. PNAS 113(40): 11366-11371.

Pietroski, P. (2018). Conjoining Meanings: Semantics without Truth Values. Oxford: Oxford University Press.

Poeppel, D. (2014). The neuroanatomic and neurophysiological infrastructure for speech and language. Current Opinion in Neurobiology 28C: 142-149. 
Pratt, J., Dawson, N., Morris, B.J., Grent-'t-Jong, T., Roux, F., \& Uhlhaas, P.J. (2017). Thalamo-cortical communication, glutamatergic neurotransmission and neural oscillations: a unique window into the origins of ScZ? Schizophrenia Research 180: 412.

Progovac, L., \& Benítez-Burraco, A. (2019). From physical aggression to verbal behavior: language evolution and self-domestication feedback loop. Frontiers in Psychology 10: 2807.

Quax, S., Jensen, O., \& Tiesinga, P. (2017). Top-down control of cortical gamma-band communication via pulvinar induced phase shifts in the alpha rhythm. PLoS Computational Biology 13(5): e1005519.

Reitter, D., Keller, F., \& Moore, J.D. (2011). A computational cognitive model of syntactic priming. Cognitive Science 35(4): 587-637.

Rogers, L.J., Vallortigara, G., \& Andrew, R.J. (2013). Divided Brains: The Biology and Behavior of Brain Asymmetries. Cambridge: Cambridge University Press.

Saalmann, Y.B. (2014). Intralaminar and medial thalamic influence on cortical synchrony, information transmission and cognition. Frontiers in Systems Neuroscience 8: 83.

Saalmann, Y. B., Pinsk, M. A., Wang, L., Li, X., \& Kastner, S. (2012). The pulvinar regulates information transmission between cortical areas based on attention demands. Science 337: 753-756.

Sambin, S., Teichmann, M., Diego Balaguer, R.D., Giavazzi, M., Sportiche, D., Schlenker, P., \& Bachoud-Lévi, A-C. (2012). The role of the striatum in sentence processing: Disentangling syntax from working memory in Huntington's disease. Neuropsychologia 50(11): 2625-2635.

Santi, A., Friederici, A. D., Makuuchi, M., \& Grodzinsky, Y. (2015). An fMRI study dissociating distance measures computed by Broca's area in movement processing: clause boundary vs. identity. Frontiers in Psychology 6: 654.

Schmahmann, J.D., \& Pandya, D.N. (1997). The cerebro-cerebellar system. International Review Neurobiology 41: 31-60. 
Schmitt, L.I., Wimmer, R.D., Nakajima, M., Happ, M., Mofakham, S., \& Halassa, M.M. (2017). Thalamic amplification of cortical connectivity sustains attentional control. Nature 545: 219-223.

Segaert, K., Mazaheri, A., \& Hagoort, P. (2018). Binding language: structuring sentences through precisely timed oscillatory mechanisms. European Journal of Neuroscience 48(7): 2651-2662.

Seger, C.A., \& Miller, E.K. (2010). Category learning in the brain. Annual Review of Neuroscience 33: 203-219.

Singer, W. (2013). Cortical dynamics revisited. Trends in Cognitive Sciences 17: 616626.

Sisterson, N.D., Carlson, A.A., Rutishauser, U., Mamelak, A.N., Flagg, M., Pouratian, N., Salimpour, Y., Anderson, W.S., Richardson, R.M. (2021). Electrocorticography during deep brain stimulation surgery: safety experience from 4 centers within the National Institute of Neurological Disorders and Stroke Research Opportunities in Human Consortium. Neurosurgery nyaa592.

Smith, K.M., \& Caplan, D.N. (2018). Communication impairment in Parkinson's disease: impact of motor and cognitive symptoms on speech and language. Brain and Language 185: 38-46.

Stocco, A., Yamasaki, B., Natalenko, R., \& Prat, C.S. (2012). Bilingual brain training: a neurobiological framework of how bilingual experience improves executive function. International Journal of Bilingualism 18(1): 67-92.

Strauss, A., Henry, M. J., Scharinger, M., \& Obleser, J. (2015). Alpha phase determines successful lexical decision in noise. Journal of Neuroscience 35(7): 32563262.

Stuss, D.T., \& Benson, D.F. (1986). The Frontal Lobes. New York: Raven.

Sweeney-Reed, C.M., Zaehle, T., Voges, J., Schmitt, F.C., Buentjen, L., Kopitzki, K., Hinrichs, H., Heinze, H-J., Rugg, M.D., Knight, R.T., \& Richardson-Klavehn, A. (2015). Thalamic theta phase alignment predicts human memory formation and anterior thalamic cross-frequency coupling. eLife 4: e07578. 
Teichmann, M., Darcy, I., Bachoud-Lévi, A-C., \& Dupoux, E. (2009). The role of the striatum in phonological processing. Evidence from early stages of Huntington's disease. Cortex 45(7): 839-849.

Teichmann, M., Dupoux, E., Kouider, S., Brugières, P., Boissé, M-F., Baudic, S., Cesaro, P., Peschanski, M., \& Bachoud-Lévi, A-C. (2005). The role of the striatum in rule application: the model of Huntington's disease at early stage. Brain 128(5): 11551167.

Teichmann, M., Rosso, C., Martini, J.B., Bloch, I., Brugières, P., Duffau, H., Lehéricy, S., \& Bachoud-Lévi, A-C. (2015). A cortical-subcortical syntax pathway linking Broca's area and the striatum. Human Brain Mapping 36: 2270-2283.

Theyel, B.B., Llano, D.A., \& Sherman, S.M. (2010). The corticothalamocortical circuit drives higher-order cortex in the mouse. Nature Neuroscience 13(1): 84-88.

Trebuchon-Da Fonseca, A., Guedj, E., Alario, F-X., Laguitton, V., Mundler, O., Chauvel, P., \& Liegeois-Chauvel, C. (2009). Brain regions underlying word finding difficulties in temporal lobe epilepsy. Brain 132: 2772-2784.

Trimmel, K., van Graan, L.A., Gonzálvez, G.G., Haag, A., Caciagli, L., Vos, S.B., Bonelli, S., Sidhu, M., Thompson, P.J., Koepp, M.J., \& Duncan, J.S. (2019). Naming $\mathrm{fMRI}$ predicts the effect of temporal lobe resection on language decline. Annals of Clinical and Translational Neurology 6(11): 2186-2196.

Ullman, M.T. (2001). The declarative/procedural model of lexicon and grammar. Journal of Psycholinguistic Research 30: 37-69.

Vallortigara, G., L.J. Rogers, L.J., \& Bisazza, A. (1999). Possible evolutionary origins of cognitive brain function. Brain Research Reviews 30: 164-175.

van Schouwenburg, M.R., den Ouden, H.E.M., \& Cools, R. (2010). The human basal ganglia modulate frontal-posterior connectivity during attention shifting. Journal of Neuroscience 30(29): 9910-9918.

Vanier, D., Sherwood, C., \& Smaers, J. (2019). Distinct patterns of hippocampal and neocortical evolution in primates. Brain, Behavior and Evolution 93: 171-181.

Volkmer, A., Wilkinson, C., Beeke, S., \& Kopelman, M. (2015). Better conversations with primary progressive aphasia (BCPPA): presenting results from the first phase of 
development of a novel communication-training package for patients and families. British Aphasiology Society Biennial International Conference. 9-11 September.

Volkmer, A., Spector, A., Warren, J.D., \& Beeke, S. (2015). Speech and language therapy for primary progressive aphasia: referral patterns and barriers to service provision across the UK. Dementia 19(5): 1349-1363.

Wahl, M., Marzinzik, F., Friederici, A.D., Hahne, A., Kupsch, A., Schneider, G.H., Saddy, D., Curio, G., \& Klostermann, F. (2008). The human thalamus processes syntactic and semantic language violations. Neuron 59: 695-707.

Wilkinson, C., \& Murphy, E. (2016). Joint interventions in autism spectrum disorder: relating oscillopathies and syntactic deficits. UCL Working Papers in Linguistics 28: 17.

Wilson, B., Marslen-Wilson, W.D., \& Petkov, C.I. (2017). Conserved sequence processing in primate frontal cortex. Trends in Neurosciences 40(2): 72-82.

Wilson, S.M., Galantucci, S., Tartaglia, M.C., Rising, K., Patterson, D.K., Henry, M.L., Ogar, J.M., DeLeon, J., Miller, B.L., \& Gorno-Tempini, M.L. (2011). Syntactic processing depends on dorsal language tracts. Neuron 72: 397-403.

Wilson, S.M., Molnar-Szakacs, I., \& lacoboni, M. (2008). Beyond superior temporal cortex: intersubject correlations in narrative speech comprehension. Cerebral Cortex 18(1): 230-242.

Wojtecki, L., Elben, S., Vesper, J., \& Schnitzler, A. (2017). The rhythm of the executive gate of speech: subthalamic low-frequency oscillations increase during verbal generation. European Journal of Neuroscience 45(9): 1200-1211.

Yeatman, J.D., \& White, A.L. (2021). Reading: the confluence of vision and language. Annual Review of Vision Science 7. https://doi.org/10.1146/annurev-vision-093019113509.

Yelnik, J., Percheron, G., \& Francois, C. (1984). A Golgi analysis of the primate globus pallidus. II. Quantitative morphology and spatial orientation of dendritic arborizations. Journal of Comparative Neurology 227(2): 200-213.

Young, J.S., Lee, A.T., \& Chang, E.F. (2021). A review of cortical and subcortical stimulation mapping for language. Neurosurgery nyaa436. 
SUBCORTICAL SYNTAX 